\title{
Latest CMS measurements of inclusive and differential top quark pair production
}

\author{
Vinicius Mikuni* \\ University of Zurich \\ E-mail: vinicius.massami.mikunidcern.ch
}

\begin{abstract}
Recent measurements performed by the CMS Collaboration regarding the inclusive and differential top quark pair production are presented. The measurements are performed using the data collected during CERN LHC Run 2, at a centre-of-mass energy of $13 \mathrm{TeV}$. Measurements are compared to state-of-the-art simulations of standard model top quark production at next-to-leading order and next-to-next-leading order for different kinematic distributions related to the top quark pair system or that uses the system as a proxy for further studies.
\end{abstract}

7th Annual Conference on Large Hadron Collider Physics - LHCP2019

20-25 May, 2019

Puebla, Mexico

*Speaker on behalf of the CMS Collaboration. 


\section{Introduction}

Top quark production measurements have always been an important pillar of the CERN LHC program. Not only is the top quark the heaviest particle described by the Standard Model (SM) but the large production of top quark pairs (t⿹弋) at the LHC energies allows an accurate comparison between theory predictions for the top production properties. With the copious amount of data collected by the CMS [1] experiment, measurements of single, double, and even triple differential cross section as a function of kinematic variables can be performed. Studies of jet substructure and underlying events also benefit from the large dataset by using the $t \bar{t}$ system as a proxy for different studies. A precise description of the t⿱t production can be used to probe next order corrections to perturbative chromodynamics (QCD) calculations and electroweak corrections. This document focuses on recent measurements provided by the CMS Collaboration for both inclusive and differential cross sections using the partial Run 2 data corresponding to an integrated luminosity of 35.9 $\mathrm{fb}^{-1}$ at $12 \mathrm{TeV}$, where the results are extrapolated to fiducial and full phase spaces and compared to state-of-the-art calculations with next-to-leading order (NLO) and next-to-next-leading order (NNLO) precision in QCD.

\section{Inclusive cross section measurement}

The inclusive production of tt pairs serve as a stringent test of the Standard Model (SM), since predictions at NNLO precision in QCD are now available [2]. The predictions rely on several different generator parameters, like the top quark mass, the strong coupling constant $\alpha_{s}$, and the parton distribution functions (PDFs) of the proton. The comparison between predictions and the measurements for different centre-of-mass energies are shown in Fig. 1 (left). The most recent inclusive cross section measurement was carried out using the dilepton decays of the $\bar{t} \bar{t}$ system at $\sqrt{s}=13 \mathrm{TeV}$ [3]. The measurement is split into different regions defined by the additional jets and the b-flavour jets multiplicity. To reduce the effect of jet energy corrections, one of the leading uncertainties, the distribution of the second highest jet $\mathrm{p}_{\mathrm{T}}$ (Figs. 2 and 3) is used as the main discriminant. This measurement reaches a standalone precision of $4 \%$ and is in good agreement with the NNLO + NNLL prediction shown in Fig. 1 (right).

\section{Differential cross section measurements}

While inclusive cross section measurements are useful to give the overall description, a differential approach can be used in order to investigate specific aspects of the theory. For instance, observables like the jet multiplicity are sensitive to high order corrections at Matrix Element (ME) level, the radiation modelling, and the parton-shower description. To compare these distributions, the cross section as a function of the jet multiplicity and other distributions are performed [5]. The results are unfolded to particle level to correct for detector effects. In Fig. 4 the normalised differential cross sections are compared to different generators. In particular, Powheg v2 [6] interfaced with Pythia8.2 [7] and MG5aMC@NLO [8] matched to PYTHIA8.2 using the FxFx prescription behave differently at different regions of the jet multiplicity spectrum.

A different distribution that has been known to be poorly described by simulations is the $\mathrm{p}_{\mathrm{T}}$ of the top quark. In Fig. 5 the measurement, unfolded to parton level, is compared to full NNLO + 

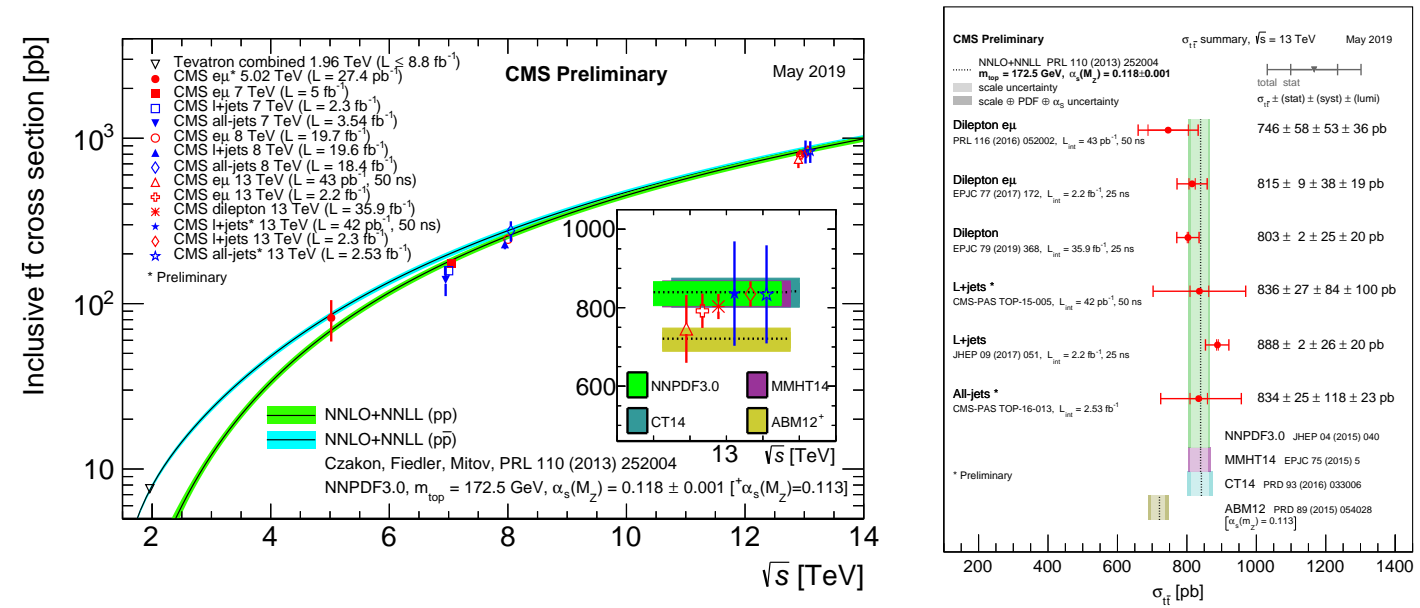

Figure 1: Left: Top quark pair cross section summary of CMS measurements in comparison with the theory calculation at NNLO+NNLL accuracy. The Tevatron measurements are also shown. Right: Top quark pair cross section summary of CMS measurements at $13 \mathrm{TeV}$ including comparison with the theory calculation at NNLO+NNLL accuracy. More information available at [4]

NNLL predictions and even beyond NNLO predictions. While the agreement does improve when considering beyond next-order corrections, the full distribution of the top quark $\mathrm{p}_{\mathrm{T}}$ is not well described by any of the current predictions.

To better characterise the features of the top quark distribution a multi-differential measurement was performed for the first time in Top-quark physics. In [9], measurements of the normalised $\mathrm{t} \overline{\mathrm{t}}$ cross section as a function of kinematic distributions related to the top and $\mathrm{t} \overline{\mathrm{t}}$ system are performed. The results are extrapolated to parton level and compared to different generators. A $\chi^{2}$ test is also performed to quantify the agreement between the measurement and predictions.

Fig. 6 shows the distribution of the top quark $\mathrm{p}_{\mathrm{T}}$ at different $\mathrm{M}(\mathrm{t} \overline{\mathrm{t}})$ regions. Comparisons with different generators confirm what was observed in [5] and give a new insight by showing that the description becomes progressively worse for higher values of $\mathrm{M}(\overline{\mathrm{t}})$.

\section{Jet substructure and underlying event measurements}

The large amount of $t \bar{t}$ events collected during Run 2 also allow measurements of other important quantities by using the $t \bar{t}$ system as a proxy. Recent CMS studies taking this approach are the investigation of jet substructure variables [10] and underlying events [11]. Jet substructure variables are important to derive different simulation tunes and to study the differences between the distributions of gluon and quark initiated jets, where the latter can be further divided into different flavour components. Fig. 7 shows the comparison between measurement and simulations for the charged constituent multiplicity for different jet origins and flavours. Comparatively, the greatest disagreement is observed for b-jets, which could profit from a flavour specific simulation tune.

The underlying event study aims at providing measurements, extrapolated to particle level, for distributions sensitive to hadronic activities that are not attributed to particles stemming from the $\mathrm{t} \overline{\mathrm{t}}$ decays. Moreover, different schemes for colour reconnection, parton-shower description, and 

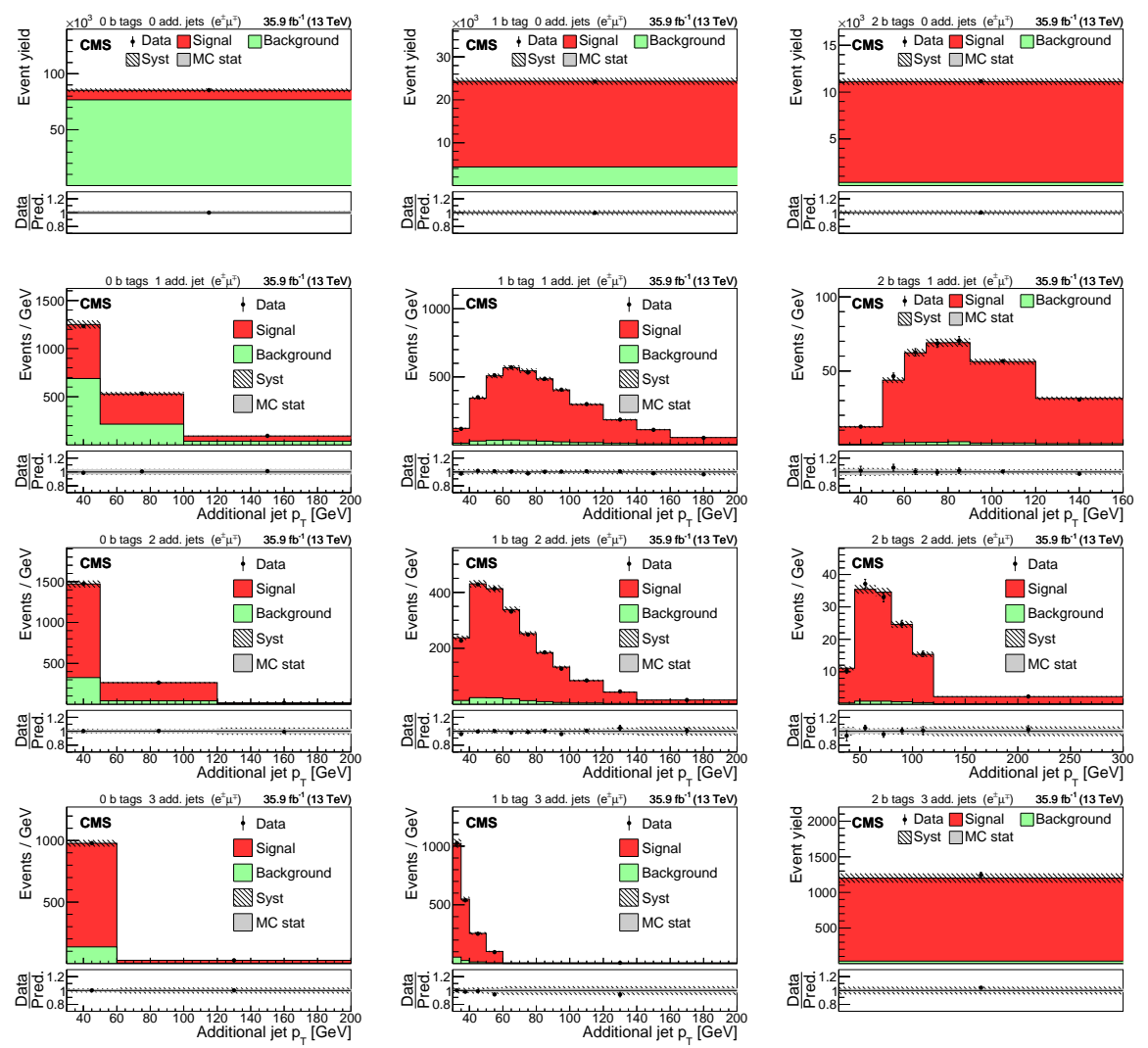

Figure 2: Distributions in the e $\mu$ channel after the fit to the data from [3]. In the left column events with zero or three or more b-tagged jets are shown. The middle (right) column shows events with exactly one (two) b-tagged jets. Events with zero, one, two, or three or more additional non-b-tagged jets are shown in the first, second, third, and fourth row, respectively. The hatched bands correspond to the total uncertainty in the sum of the predicted yields including all correlations. The ratios of the data to the sum of the simulated yields after the fit are shown in the lower panel of each figure. Here, the solid gray band represents the contribution of the statistical uncertainty in the MC simulation.

multi-parton (MPI) scattering effects can be probed. Fig. 8 shows the hadronic activity distribution for different variations of the generator parameters. Comparing with the measurement, scenarios using lower values of $\left.\alpha_{S}^{F S R}\left(m_{Z}\right)\right)$ are favoured. One can also conclude that considering MPI effects to the simulation largely improves the description of the generators.

\section{Summary}

With the large dataset collected during the Run 2 of the LHC, the CMS Collaboration has performed measurements of the inclusive and differential cross sections of the $t \bar{t}$ system, providing insight to different stages of the event generation. The different measurements are important to further improve the description of different simulations, while characterising discrepancies observed.

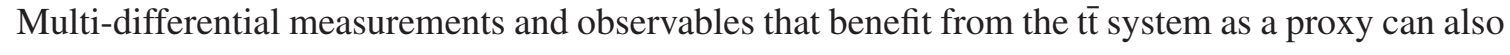
be further improved by exploiting new analysis techniques and the full Run 2 dataset. 

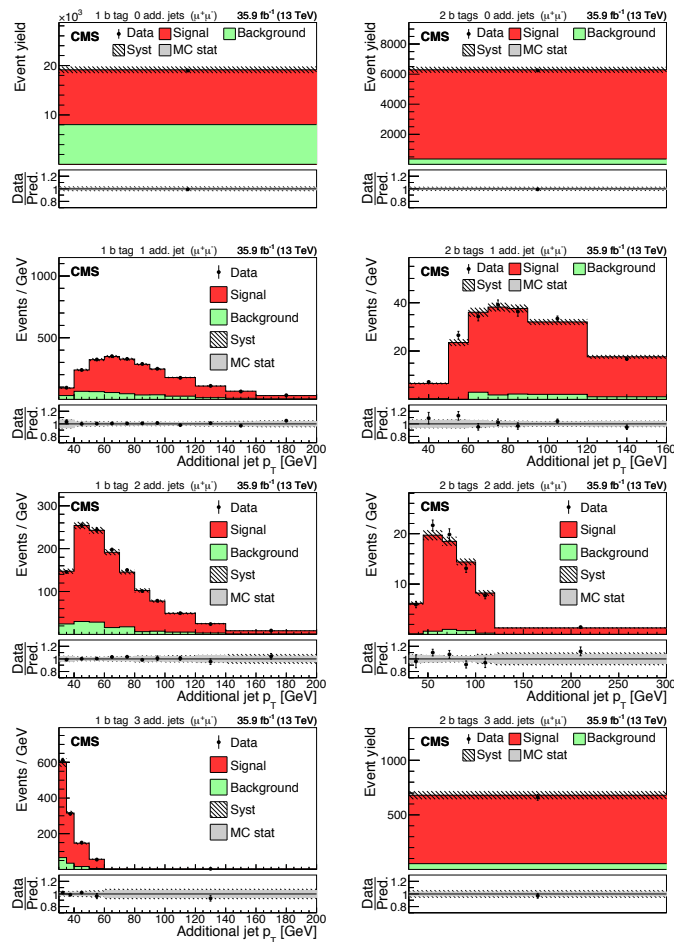
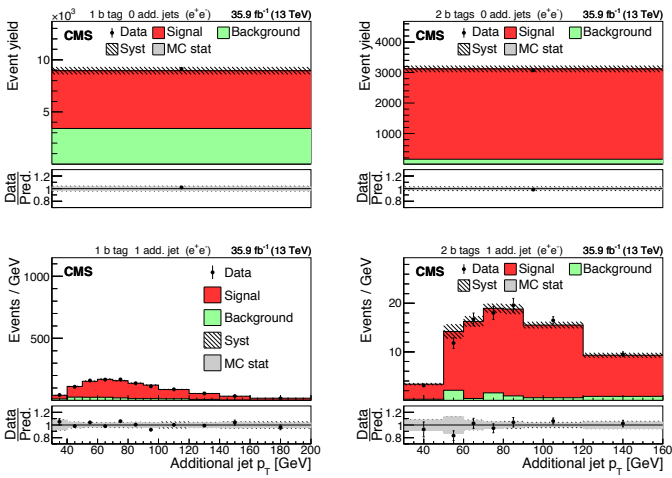
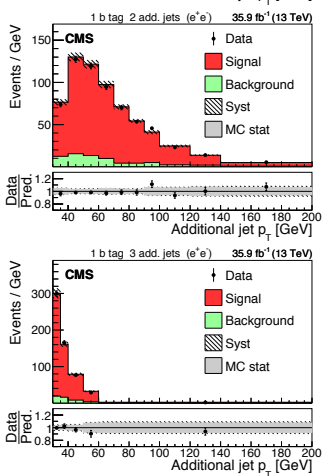
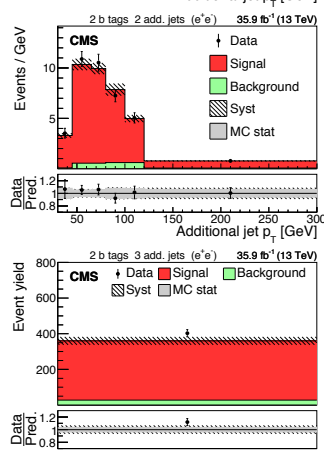

Figure 3: Distributions in the $\mu \mu$ channel (left) and ee channel (right) after the fit to the data from [3]. Further details are described in the caption of Fig. 2.
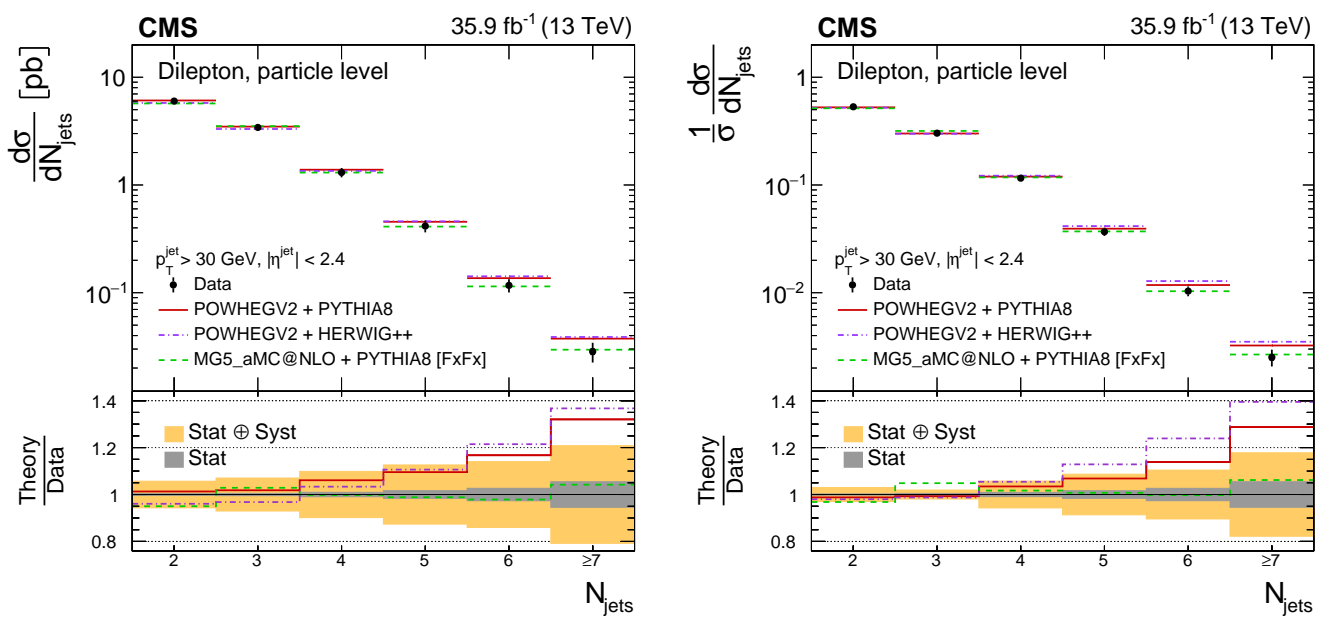

Figure 4: The differential $\bar{t} \bar{t}$ production cross sections as a function of $N_{\text {jets }}$ from [5] in a fiducial phase space at the particle level are shown for the data (points) and the MC predictions (lines). The vertical lines on the filled circles and other points indicate the total uncertainty in the data and theoretical predictions, respectively. The left and right plots correspond to absolute and normalised measurements, respectively. 

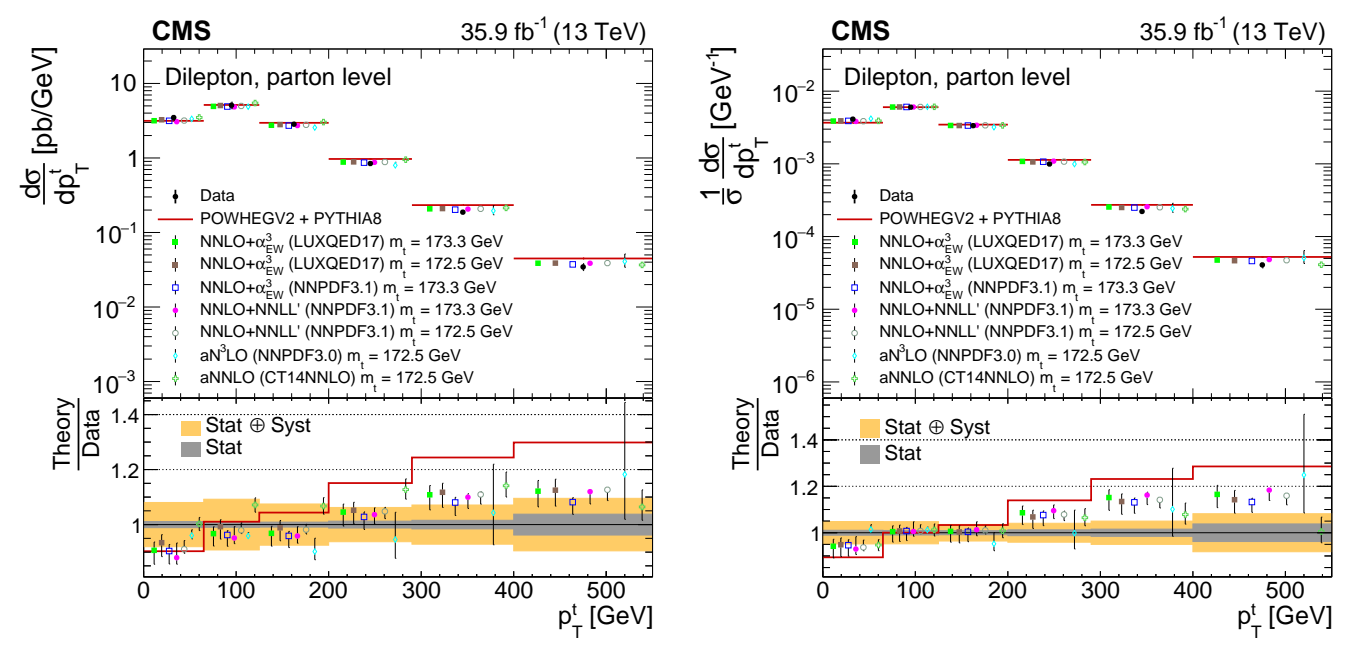

Figure 5: The differential $\bar{t} \bar{t}$ production cross sections as a function of $\mathrm{p}_{\mathrm{T}}$ from [5] are shown for the data (filled circles), the theoretical predictions with beyond-NLO precision (other points) and the prediction from POWHEG+PYTHIA (solid line). Further details are described in the caption of Fig. 4.

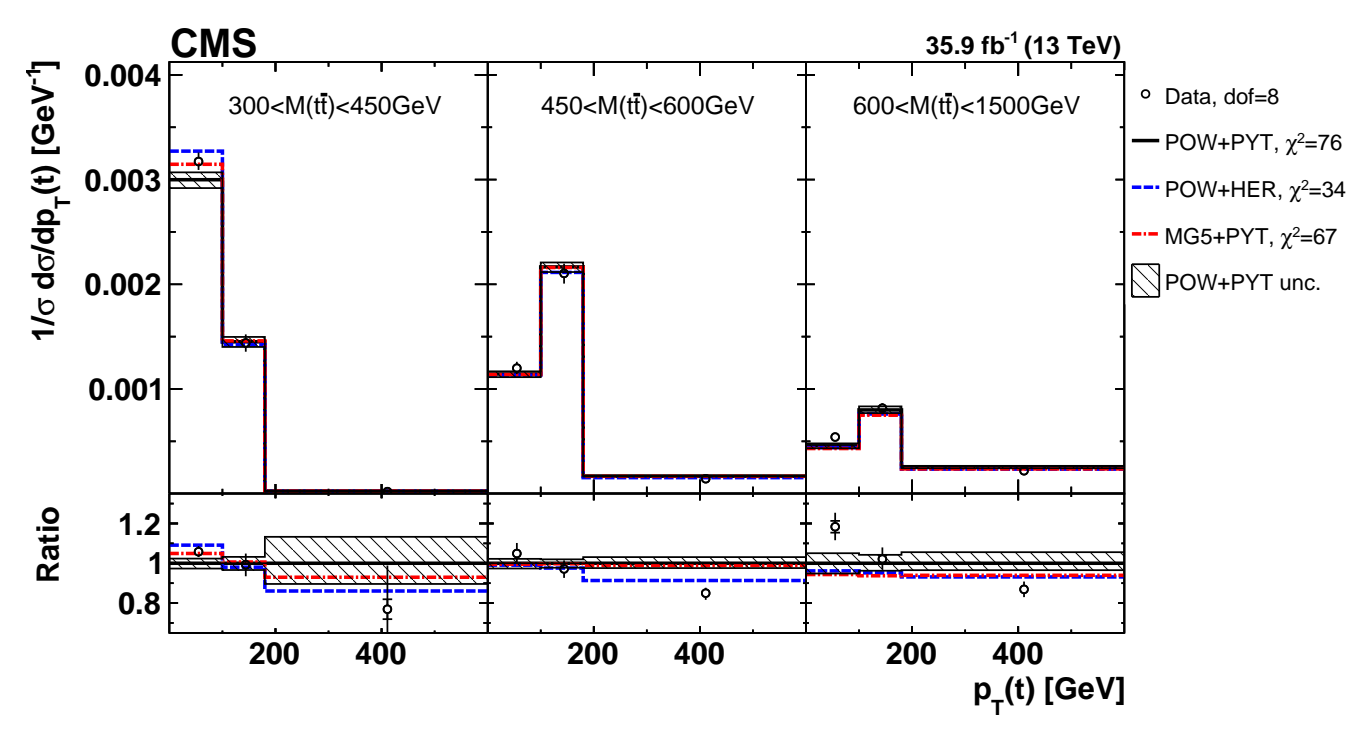

Figure 6: Comparison of the measured [ $\left.\mathrm{M}(\mathrm{t} \overline{\mathrm{t}}), \mathrm{p}_{\mathrm{T}}(\mathrm{t})\right]$ cross sections from [5] to the theoretical predictions calculated using MC event generators. The inner vertical bars on the data points represent the statistical uncertainties and the full bars include also the systematic uncertainties added in quadrature. For each MC model, values of $\chi^{2}$ which take into account the bin-to-bin correlations and dof for the comparison with the data are reported. The hatched regions correspond to the theoretical uncertainties in POWHEG + PYTHIA. In the lower panel, the ratios of the data and other simulations to the 'POW+PYT' predictions are shown. 

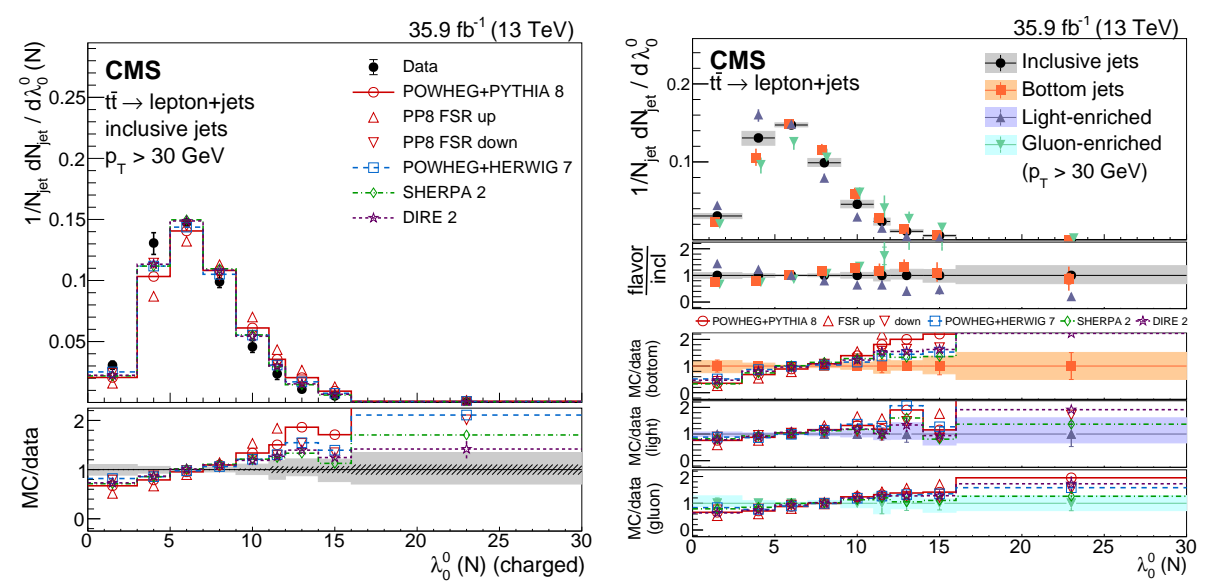

Figure 7: Left: Charged particle multiplicity $\lambda_{0}^{0}(\mathrm{~N})$ normalised and unfolded to the particle level, for inclusive jets from [10]. Data (points) are compared to different MC predictions (upper), and as MC/data ratios (lower). The hatched and shaded bands represent the statistical and total uncertainties, respectively. Right: Distribution of the charged multiplicity, unfolded to the particle level, for jets of different flavors. The second panel shows the corresponding ratios of the different flavors over the inclusive jets data. The sub-panel shows the ratios of the different MC predictions over the bottom, light-quark-enriched, and gluonenriched jet data.
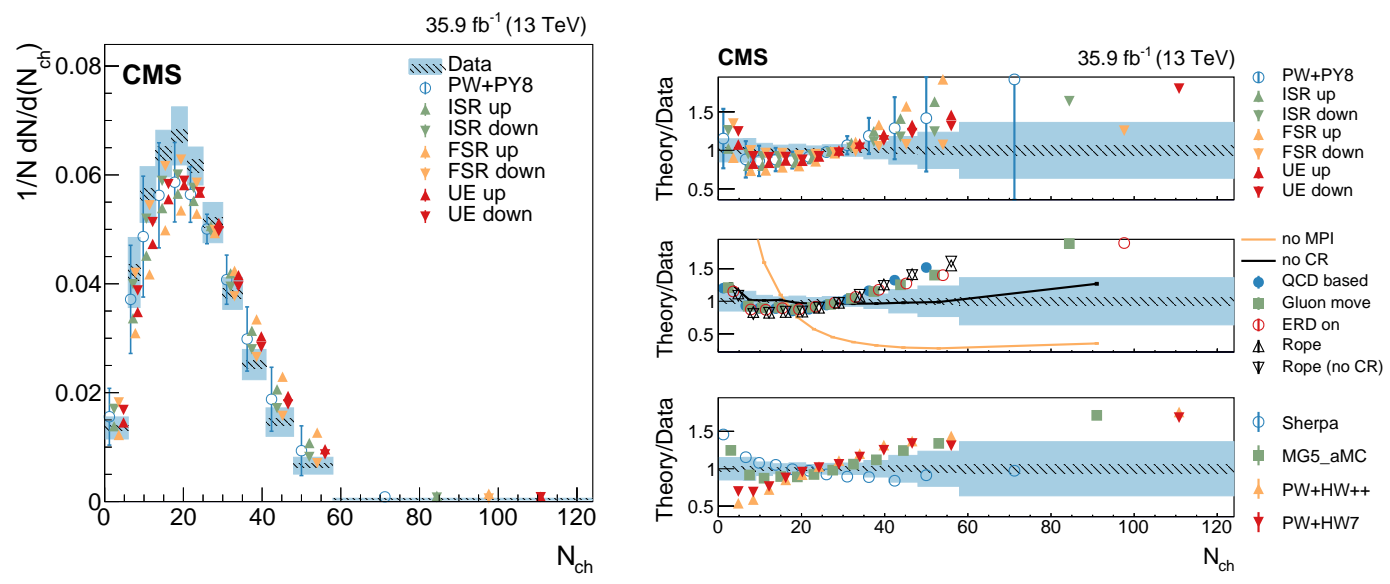

Figure 8: The normalised differential cross section as a function of $\mathrm{N}_{c h}$ is shown from [11]. The data (coloured boxes) are compared to the nominal POWHEG+PYTHIA 8 predictions and to the expectations obtained from varied $\alpha_{S}^{I S R}\left(m_{Z}\right)$ or $\left.\alpha_{S}^{F S R}\left(m_{Z}\right)\right)$ POWHEG+PYTHIA8 setups (markers). The different panels on the lower display show the ratio between each model tested (see text) and the data. In both cases the shaded (hatched) band represents the total (statistical) uncertainty of the data, while the error bars represent either the total uncertainty of the POWHEG+PYTHIA8 setup, computed as described in the text, or the statistical uncertainty of the other MC simulation setups. 


\section{References}

[1] CMS Collaboration, The CMS Experiment at the CERN LHC, JINST 3 (2008) S08004.

[2] M. Czakon, P. Fiedler and A. Mitov, Total Top-Quark Pair-Production Cross Section at Hadron Colliders Through $O\left(\alpha \frac{4}{S}\right)$, Phys. Rev. Lett. 110 (2013) 252004 [1303. 6254].

[3] CMS Collaboration, Measurement of the $\mathrm{t} \overline{\mathrm{t}}$ production cross section, the top quark mass, and the strong coupling constant using dilepton events in pp collisions at $\sqrt{s}=13 \mathrm{TeV}$, Eur. Phys. J. C79 (2019) 368 [1812.10505].

[4] “CMS Top Quark Physics Summary Figures.” https://twiki.cern.ch/twiki/bin/view/ CMSPublic/PhysicsResults TOP SummaryFigures, May, 2019.

[5] CMS Collaboration, Measurements of $\mathrm{t} \overline{\mathrm{t}}$ differential cross sections in proton-proton collisions at $\sqrt{s}=$ $13 \mathrm{TeV}$ using events containing two leptons, JHEP 02 (2019) 149 [1811. 06625 ].

[6] S. Frixione, P. Nason and G. Ridolfi, A Positive-weight next-to-leading-order Monte Carlo for heavy flavour hadroproduction, JHEP 09 (2007) 126 [0 707 . 3088].

[7] T. Sjöstrand, S. Ask, J. R. Christiansen, R. Corke, N. Desai, P. Ilten et al., An Introduction to PYTHIA 8.2, Comput. Phys. Commun. 191 (2015) 159 [1410.3012].

[8] J. Alwall, R. Frederix, S. Frixione, V. Hirschi, F. Maltoni, O. Mattelaer et al., The automated computation of tree-level and next-to-leading order differential cross sections, and their matching to parton shower simulations, JHEP 07 (2014) 079 [1405.0301].

[9] CMS Collaboration, Measurement of $\mathrm{t} \overline{\mathrm{t}}$ normalised multi-differential cross sections in pp collisions at $\sqrt{s}=13 \mathrm{TeV}$, and simultaneous determination of the strong coupling strength, top quark pole mass, and parton distribution functions, Submitted to: Eur. Phys. J. (2019) [1904.05237].

[10] CMS Collaboration, Measurement of jet substructure observables in $\mathrm{t} \overline{\mathrm{t}}$ events from proton-proton collisions at $\sqrt{s}=13$ TeV, Phys. Rev. D98 (2018) 092014 [1808.07340].

[11] CMS Collaboration, Study of the underlying event in top quark pair production in pp collisions at 13 TeV , Eur. Phys. J. C79 (2019) 123 [1807.02810]. 